\title{
Pectoral muscle detection in mammograms based on polar coordinates and the shortest path
}

\author{
Jaime S. Cardoso, Member, IEEE, Inês Domingues, Student Member, IEEE, Igor Amaral, \\ Inês Moreira, Pedro Passarinho, João Santa Comba, Ricardo Correia, Maria J. Cardoso
}

\begin{abstract}
The automatic detection and segmentation of the pectoral muscle in the medio-lateral oblique view of mammograms is essential for further analysis of breast anormalies. However, it is still a very difficult task since the sizes, shapes and intensity contrasts of pectoral muscles change greatly from image to image. In this paper, an algorithm based on the shortest path on a graph is proposed to automatically detect the pectoral muscle contour. To overcome the difficulties of searching for the path between a lateral and the top margins of the image, this is first transformed, using polar coordinates. In the transformed image, the muscle boundary in amongst the shortest paths between the top and the bottom rows. A comprehensive comparison with manually-drawn contours reveals the strength of the proposed method.
\end{abstract}

\section{INTRODUCTION}

In spite of the progresses in other breast imaging modalities (magnetic resonance imaging, etc.), mammography is the only screening modality proven to detect early stage breast cancer.

The pectoral muscle represents a predominant density region in most medio-lateral oblique (MLO) views of mammograms, and can affect the results of image processing methods. Intensity-based methods, for example, can present poor performance when applied to differentiate dense structures such as the fibro-glandular disc or small suspicious masses, since the pectoral muscle appears at approximately the same density as the dense tissues of interest in the image. The inclusion of the pectoral muscle in the image data being processed could also bias the detection procedures. Another important need to identify the pectoral muscle lies in the possibility that the local information of its edge, along with an internal analysis of its region, may be used to identify the presence of abnormal axillary lymph nodes, which may be the only manifestation of occult breast carcinoma. [1]

The current image evaluation criteria for the mammographic presentation of the pectoral muscle on the MLO view of the breast recommends that the inferior aspect of the pectoral muscle reaches the level of the nipple. However, many MLO mammograms fail this quality criterion of the image evaluation systems; in [2] it was observed that $75.5 \%$

This work was funded by the Portuguese Innovation Agency (ADI) through project QREN reference 3472 "Semantic PACS". J. S. Cardoso, I. Domingues and I. Amaral are with INESC Porto, Faculdade de Engenharia, Universidade do Porto, Portugal. jaime.cardoso@inescporto.pt, inesdomingues@gmail.com, igor.amaral@gmail.com

I. Moreira, R. Correia and M. J. Cardoso are with Faculdade de Medicina, Universidade do Porto, Portugal. ines.c.moreiraegmail.com, ricardo.jc.correia@gmail.com, mjcard@med.up.pt

P. Passarinho, J. Santa Comba are with Emílio Azevedo Campos, Portugal. \{pedro.passarinho, joao.comba\}eacampos.pt of the mammograms failed the criterion. In some mammograms the pectoral muscle is not present at all. This wide variability in the position of the muscle contour, together with the similarity between muscle and breast tissues make the detection a difficult task.

\section{A. Related works}

One of the most used pectoral muscle segmentation algorithms is the method proposed by Ferrari et al. [3] based on the Hough transform. The main problem with this approach is that the pectoral muscle is approximated by a line. These methods give poor results when the pectoral muscle contour is a curve. For this reason, the same team proposed another method [1] based on Gabor wavelets. In [4], the pectoral muscle was once again approximated by a straight line, but this line was further adjusted through surface smoothing and edge detection.

Ma et al. [5] described two image segmentation methods: one based on adaptive pyramids and other based on minimum spanning trees. The article [6] chose the longest straight line in Radon-domain as an approximation to the pectoral muscle localization. The problem with this work is twofold: the simplification of using a line and the use of a private database, so the results cannot be compared with other publications. Camilus and co-workers [7] used a graph cut method followed by Bezier curve smoothing. Recently, an isocontour map methodology was proposed [8]. Finally, a discrete time Markov chain and an active contour model were adopted in [9] for muscle detection.

Although the long list of related works, almost none addresses the problem of deciding if the muscle contour is present or not in the mammogram. Moreover, some works assume user input, either in the form of a region of interest or as a set of points in the contour. Finally, with the increased use of digital mammograms, and with its inherent higher quality, simpler approaches could be more adequate.

\section{PeCtoral MUSCle Detection By Shortest PATH}

Since in the proposed method the image grid is considered as a graph with pixels as nodes and edges connecting neighbouring pixels, we start by introducing some graph concepts.

\section{A. Background knowledge}

A graph $G=(V, A)$ is composed of two sets $V$ and $A$. $V$ is the set of nodes, and $A$ the set of $\operatorname{arcs}(p, q), p, q \in V$. The graph is weighted if a weight $w(p, q)$ is associated to 
each arc. The weight of each arc, $w(p, q)$, is a function of pixels values and pixels relative positions. A path from vertex (pixel) $v_{1}$ to vertex (pixel) $v_{n}$ is a list of unique vertices $v_{1}, v_{2}, \ldots, v_{n}$, with $v_{i-1}$ and $v_{i}$ corresponding to neighbour pixels. The total cost of a path is the sum of each arc weight in the path $\sum_{i=2}^{n} w\left(v_{i-1}, v_{i}\right)$.

A path from a source vertex $v$ to a target vertex $u$ is said to be the shortest path if its total cost is minimum among all $v$-to- $u$ paths. The distance between a source vertex $v$ and a target vertex $u$ on a graph, $d(v, u)$, is the total cost of the shortest path between $v$ and $u$.

A path from a source vertex $v$ to a sub-graph $\Omega$ is said to be the shortest path between $v$ and $\Omega$ if its total cost is minimum among all $v$-to- $u \in \Omega$ paths. The distance from a node $v$ to a sub-graph $\Omega, d(v, \Omega)$, is the total cost of the shortest path between $v$ and $\Omega$ :

$$
d(v, \Omega)=\min _{u \in \Omega} d(v, u) .
$$

A path from a sub-graph $\Omega_{1}$ to a sub-graph $\Omega_{2}$ is said to be the shortest path between $\Omega_{1}$ and $\Omega_{2}$ if its total cost is minimum among all $v \in \Omega_{1}$-to- $u \in \Omega_{2}$ paths. The distance from a sub-graph $\Omega_{1}$ to a sub-graph $\Omega_{2}, d\left(\Omega_{1}, \Omega_{2}\right)$, is the total cost of the shortest path between $\Omega_{1}$ and $\Omega_{2}$.

$$
d\left(\Omega_{1}, \Omega_{2}\right)=\min _{v \in \Omega_{1}, u \in \Omega_{2}} d(v, u) .
$$

In graph theory, the shortest-path problem seeks the shortest path connecting two nodes; efficient algorithms are available to solve this problem, such as the well-known Dijkstra algorithm [10].

\section{B. Base Algorithm for Pectoral Muscle Detection}

We will assume in the following that the image of the MLO mammogram has already been mirrored, if necessary, using information from the DICOM header or with a trivial histogram analysis (by comparing the average intensity level of the right half with the left half), so that the muscle boundary, if present, goes from the top margin to the left margin of the image.

Intuitively, the muscle boundary manifests itself as a change in the gray-level values of the pixels, giving origin to an edge in the resulting image. Therefore, we can argue that the muscle boundary corresponds to a path through edge pixels. If paths through edges pixels are favored with the appropriate weight in the graph, the muscle boundary is a low-cost path between the left and the top margins.

A difficulty with searching for the shortest paths (shortest in the sense on minimizing the cost of the path) between the top row and left column is that small paths, near the top-left corner are naturally favoured. To overcome this challenge, we propose to pre-process the image, adopting the polar coordinates, see Fig. 1. We assume that the origin of the coordinates is in the top-left corner. Note that the left column is mapped to the bottom row (corresponding to an angle of $\pi / 2$ rads) and the top row stays in the same position. In this new coordinate-system, the path to search for is now between the top and bottom rows.

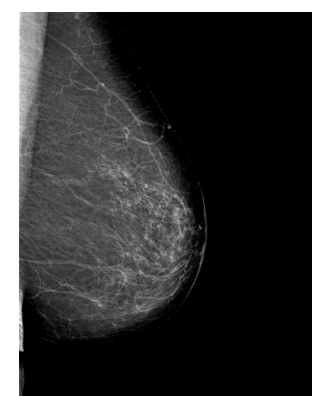

(a) Original image.

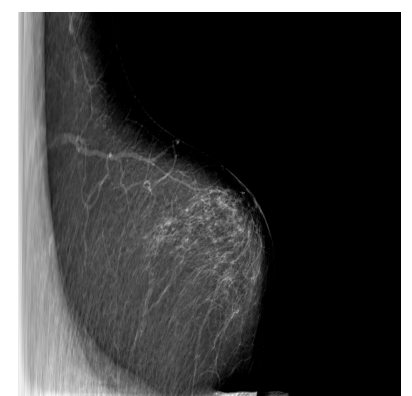

(b) Image after polar transformation. The horizontal axis represents the radius and the vertical axis the angle, from 0 to 90 degrees.

Fig. 1. Mammogram pre-processing.

In the transformed image, one may assume the simplifying assumption that the vertical paths do not zigzag back and forth, up and down. Therefore, the search may be restricted among connected paths containing one, and only one, pixel in each row between the two end-rows. Formally, let I be an $N_{1} \times N_{2}$ image (after polar coordinate transform) with $N_{1}$ columns and $N_{2}$ rows; define a vertical path to be

$$
\mathbf{s}=\{(x(y), y)\}_{y=\Omega_{1}}^{\Omega_{2}}, \text { s.t. } \forall y|x(y)-x(y-1)| \leq 1,
$$

where $x$ is a mapping $x:\left[\Omega_{1}, \cdots, \Omega_{2}\right] \rightarrow\left[1, \cdots, N_{1}\right]$. That is, a vertical path is an 8-connected path of pixels in the image from $\Omega_{1}$ to $\Omega_{2}$, containing one, and only one, pixel in each row of the image.

The optimal vertical path that minimizes this cost can be found using dynamic programming (instead of resorting to a more complex algorithm for the generic shortest path problem) [11], [12]. The first step is to traverse the image from the first to the last row and compute the cumulative minimum cost $\mathrm{C}$ for each entry $(i, j)$ :

$C(i, j)=\min \left\{\begin{array}{rl}C(i-1, j-1) & +w\left(p_{i-1, j-1} ; p_{i, j}\right) \\ C(i, j-1) & +w\left(p_{i, j-1} ; p_{i, j}\right) \\ C(i+1, j-1) & +w\left(p_{i+1, j-1} ; p_{i, j}\right)\end{array}\right.$,

where $w\left(p_{i, j} ; p_{l, m}\right)$ represents the weight of the arc incident with pixels at positions $(i, j)$ and $(l, m)$. At the end of this process,

$$
\min _{i \in\left\{1, \cdots, N_{1}\right\}} C\left(i, N_{2}\right)
$$

indicates the end of the minimal connected path. Hence, in the second step, one backtracks from this minimum entry on $C$ to find the optimal vertical path.

\section{Proposed Algorithm}

The proposed algorithm ${ }^{1}$ can be implemented as a sequence of a few high-level operations, as presented in Listing 1.

The main processing encompasses:

\footnotetext{
${ }^{1}$ The Matlab source code is available upon request to the authors.
} 


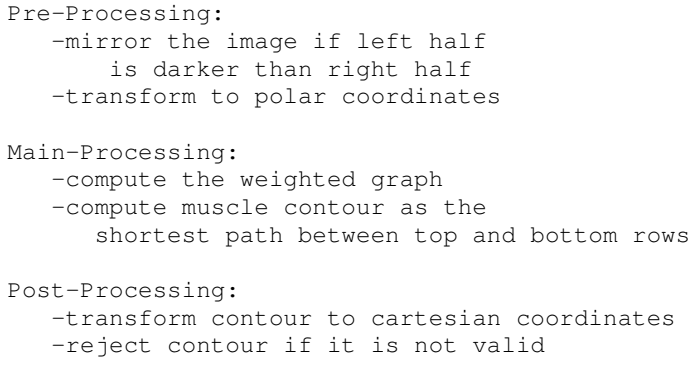

Listing 1: Main operations of the proposed method.

- A gradient computation of the original image. In a broader view, this can be replaced by any feature extraction process that emphasizes the pixels we are seeking for (pixels on the pectoral muscle boundary).

- Consider the gradient image as a weighted graph with pixels as nodes and edges connecting neighbouring pixels. Assign to an arc an weight $w$ determined by the gradient values of the two incident pixels.

In this work, the weight of the arc connecting 4-neighbour pixels $p$ and $q$ was expressed as an exponential law:

$$
f(g)=f_{\ell}+\left(f_{h}-f_{\ell}\right) \frac{\exp (\beta(255-g))-1}{\exp (\beta 255)-1},
$$

with $f_{\ell}, f_{h}, \beta \in \mathbf{R}$ and $g$ is the minimum of the gradient computed on the two incident pixels. For 8-neighbour pixels the weight was set to $\sqrt{2}$ times that value. The parameters $f_{\ell}$ and $f_{h}$ were fixed at $f_{\ell}=2$ and $f_{h}=32 ; \beta$ was experimentally tuned using a grid search method, yielding $\beta=0.025$.

The gradient model adopted in the experiments reported shortly is based on the Prewitt operator. The Prewitt operator is applied on the $x$ and $y$ directions; from the computed values, $G_{x}$ and $G_{y}$, the magnitude of the gradient is estimated as $g=\sqrt{G_{x}^{2}+G_{y}^{2}}$.

Since the pectoral muscle may not be present in some MLO images and the main processing step of our approach always outputs a predicted boundary, it is necessary to validate if the detected path should be accepted or not as a muscle boundary.

The validation rule, in the cartesian coordinate representation, assumes that a muscle boundary runs 'more or less monotonously' from the top margin to the left margin. In particular it is not expected that the path deviates a lot to the right of the end point on the top row; likewise, the path should also not go a lot below the endpoint on the left margin. Therefore, if the column of rightmost point in the contour is more than $k$ times the column of the end point on the top row or the row of the lowest point is more than $k$ times the row of the end point on the left column, the contour is rejected and the mammogram is assumed to be without a muscle boundary. In the experimental setup, $k$ was set at 1.2.

\section{RESULTS}

The methodology proposed in this paper was assessed on a set of 150 mammograms. One hundred mammograms were collected at Hospital S. João (HSJ), Porto, Portugal. These mammograms are Full Field Digital Mammography (FFDM), already preprocessed by the acquisition equipment for display purposes. Our detection algorithm was also tested on 50 images from the Digital Database for Screening Mammography (DDSM) (University of South Florida, 2001). In order to evaluate the performance of the proposed method, an experienced radiologist was asked to manually mark the contours on the 150 mammograms. For evaluation purposes, when no contour was present in the mammogram, the reference contains only a single point at position $(0,0)$.

Independently of the original size, the image in polar coordinates was kept at a constant $1024 \times 1024$ size. This improves the computational performance of the implementation of the software, without degrading the quality of the final result.

To evaluate the quality of the muscle boundary detection algorithm we conducted a complete objective evaluation, based on the hausdorff and the average distances to compare two contours. The hausdorff distance is defined as

$$
H(A, B)=\max (h(A, B), h(B, A)),
$$

where $\mathrm{A}$ and $\mathrm{B}$ represent the sets of the pixels in the reference muscle contour and the segmented muscle contour respectively, and

$$
h(A, B)=\max _{a \in A} \min _{b \in B}\|a-b\|
$$

and $\|$.$\| is the Euclidean distance. The motivation for using$ this metric is that is represents the "worst case scenario".

Fig. 2 shows the evolution of the error when estimating the position of the muscle contour. The error in pixels (in the cartesian coordinate system) was normalized by the size of the diagonal of the original image. Table I summarizes the results.

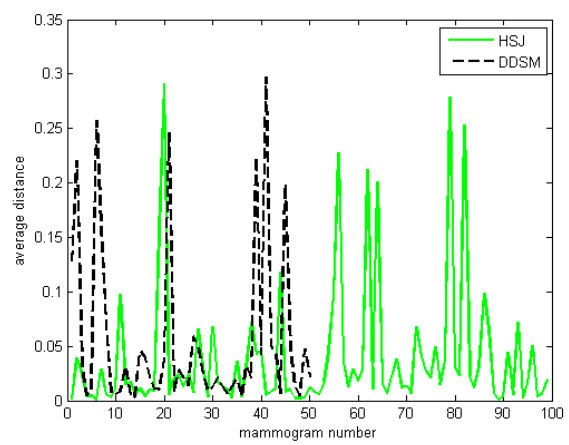

Fig. 2. Error (normalized by the diagonal of the image) in the position of the muscle boundary of the proposed method as measured by the average distance over 50 DDSM mammograms and 100 HSJ mammograms.

Fig. 3 shows some of the images in which the algorithm worked satisfactorily. Some of the unsuccessful cases, displayed in Fig. 4, bring to light the limitations of the current state of the proposed approach.

We observed a propensity to miss the part of the contour next to the left margin, where the boundary is usually more 
TABLE I

OVERALL RESULTS FOR OVER THE TWO DATABASES, IN THE POSITION OF THE MUSCLE BOUNDARY.

\begin{tabular}{|c||c|c|}
\hline Database & $\begin{array}{c}\text { Hausdorff Distance } \\
\text { (normalized) }\end{array}$ & $\begin{array}{c}\text { Mean Distance } \\
\text { (normalized) }\end{array}$ \\
\hline \hline DDSM & 0.1387 & 0.0545 \\
\hline HSJ & 0.1426 & 0.0387 \\
\hline
\end{tabular}

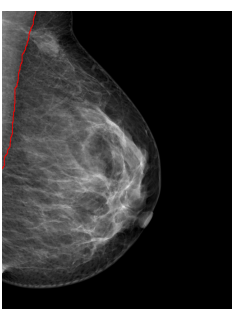

(a) Image HSJ \#05

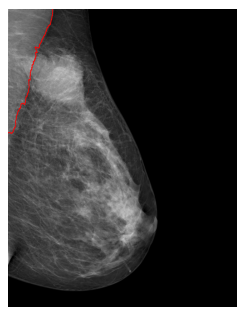

(b) Image DDSM \#22.

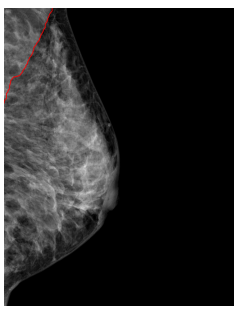

(c) Image HSJ \#35.
Fig. 3. Selected successful results.

diffuse. Moreover, when there are multiple strong edges, the algorithm may pick the wrong one. Finally, and a little surprisingly, the results for the digital mammograms are not clearly superior to the results over the screen film mammograms.

\section{CONCLUSION}

In the proposed framework, the delimitation of the pectoral muscle in a mammogram is formulated as a problem of finding the shortest path between two regions in a graph, after conveniently modelling the image as an weighted graph.

A traditional difficulty hampering the application methodologies based on the shortest path is the definition of the endpoints, often manually placed. We overcame that challenge by computing the shortest path between two whole margins of the image on a transformed coordinate system. Despite the simplicity and few assumptions of the method, the results are quite promising.

In opposition to many previous works reported in the literature, we also do not assume that the muscle boundary is necessarily present in the mammogram. This is especially important is screening mammograms. Although the quality criteria for MLO mammograms dictates that the inferior aspect of the pectoral muscle reaches the level of the nipple, that is often not achieved, subsisting a wide variability on the position of the muscle, including its complete absence.

Another, more involved solution, is to learn the endpoints using supervised learning methodologies before computing the shortest path between the now known endpoints [13]. Although learning automatically the endpoints has the potential to get better results, there is also the danger that the model learnt to predict the endpoints from a training set of images acquired with a given equipment will not generalize well for equipments from different manufacturers. The model proposed in this article, by depending much less on prior

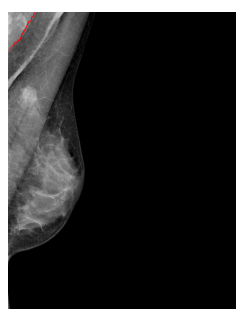

(a) Image HSJ \#39.

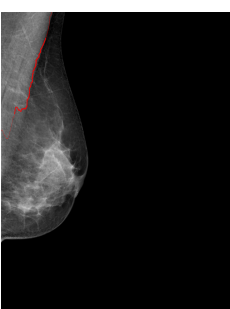

(b) Image HSJ \#45.

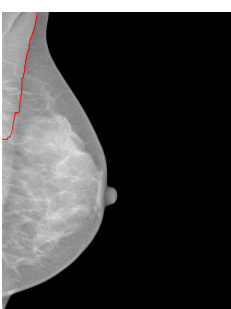

(c) Image HSJ \#98.
Fig. 4. Selected poor results.

knowledge, will not likely suffer from that problem. Further investigation is necessary to assess the tradeoffs involved.

Future work will focus on the investigation of new features to replace the prewitt-based gradient, capturing better the distinction between muscle and breast tissue. We plan to incorporate in the weight function of the graph, local and global information. The validation rule to accept if a detected path is or not a muscle boundary also needs to be refined.

\section{REFERENCES}

[1] R. J. Ferrari, R. M. Rangayyan, J. E. L. Desautels, R. A. Borges, and A. F. Frère, "Automatic identification of the pectoral muscle in mammograms," IEEE Trans on Medical Imaging, vol. 23, no. 2, pp. 232-245, 2004.

[2] K. Bentley, A. Poulos, and M. Rickard, "Mammography image quality: Analysis of evaluation criteria using pectoral muscle presentation,' Radiography, vol. 14, no. 3, pp. 189-194, 2008.

[3] R. J. Ferrari, R. M. Rangayyan, J. E. L. Desautels, R. A. Borges, and A. F. Frère, "Segmentation of mammograms: Identification of the skin-air boundary, pectoral muscle, and fibro-glandular disc," in Proc of the 5th International Workshop on Digital Mammography, 2000 pp. 573-579.

[4] S. Kwok, R. Chandrasekhar, and Y. Attikiouzel, "Automatic pectoral muscle segmentation on mammograms by straight line estimation and cliff detection," in Intelligent Information Systems Conference, The Seventh Australian and New Zealand 2001, 2001, pp. 67-72.

[5] F. Ma, M. Bajger, J. P. Slavotinek, and M. J. Bottema, "Two graph theory based methods for identifying the pectoral muscle in mammograms," Pattern Recognition, vol. 40, no. 9, pp. 2592-2602, 2007.

[6] S. K. Kinoshita, P. M. Azevedo-Marques, J. A. R. R. R. Pereira Jr, and R. M. Rangayyan, "Radon-domain detection of the nipple and the pectoral muscle in mammograms," Journal Digital Imaging, vol. 21, no. 1, pp. 37-49, 2008.

[7] K. S. Camilus, V. K. Govindan, and P. S. Sathidevi, "Computeraided identification of the pectoral muscle in digitized mammograms,' Journal Digital Imaging, 2009.

[8] B. W. Hong and B. S. Sohn, "Segmentation of regions of interest in mammograms in a topographic approach," IEEE Trans Inf Technol Biomed, vol. 14, no. 1, pp. 129-139, 2010.

[9] L. Wang, M. Zhu, L. ping Deng, and X. Yuan, "Automatic pectoral muscle boundary detection in mammograms based on markov chain and active contour model," Journal of Zhejiang University - Science $C$, vol. 11, no. 2, pp. 111-118, 2010.

[10] E. W. Dijkstra, "A note on two problems in connexion with graphs," Numerische Mathematik, vol. 1, pp. 269-271, 1959.

[11] J. S. Cardoso, A. Capela, A. Rebelo, C. Guedes, and J. F. P. da Costa, "Staff detection with stable paths," IEEE Trans Pattern Analysis Machine Intelligence, vol. 31, pp. 1134-1139, 2009.

[12] H. P. Oliveira and J. S. Cardoso, "Image retargeting using stable paths," in Proc of the Fourth International Conference on Computer Vision Theory and Applications, vol. 2, 2009, pp. 40-47.

[13] I. Domingues, J. S. Cardoso, I. Amaral, I. Moreira, P. Passarinho, J. S. Comba, R. Correia, and M. J. Cardoso, "Pectoral muscle detection in mammograms based on the shortest path with endpoints learnt by svms (submitted)," in Proc of the 32nd Annual International Conference of the IEEE Engineering in Medicine and Biology Society, 2010. 